\title{
Comparison of Catch-Curve Methods for Estimation of Mortality
}

\author{
A. L. JENSEN \\ School of Natural Resources, University of Michigan \\ Ann Arbor, Michigan 48109
}

\begin{abstract}
Several methods are available to estimate mortality from catch curves. These methods include least squares, a maximum-likelihood method developed by Chapman and Robson, and a "simple" estimator proposed by Ssentongo and Larkin. All three of these methods are based on similar models for mortality, but data requirements are different, and they can give considerably different estimates of mortality. These three methods are compared by applying them to catch curves for natural populations, to catch curves generated by a deterministic model, and to catch curves generated by a stochastic model. In general, the least-squares estimator is the most precise under conditions encountered in assessment of fisheries, but if variation is unusually high, the maximumlikelihood estimator developed by Chapman and Robson is more precise. The "simple" estimator developed by Ssentongo and Larkin can result in serious error.
\end{abstract}

Received January 14, 1984

Accepted April 22, 1985

Catch-curve data are widely applied to estimate mortality for sport and small commercial fisheries where mark-recapture experiments and cohort analysis are not feasible. The methods most frequently applied are the maximum-likelihood (ML) estimator based on the geometric distribution, which will be denoted as ML1 (Chapman and Robson 1960; Robson and Chapman 1961); the ML estimator based on the exponential distribution, which will be denoted as ML2 (Ssentongo and Larkin 1973); and the leastsquares (LS) estimator. Methods developed by Jackson (1939) and Heincke (1913) often were applied in the past (Ricker 1958). Jackson's method is poorly defined statistically (Chapman and Robson 1960). Heincke's method is an LS estimator calculated with untransformed data and it is better to transform the data before doing the calculations (Seber 1973). The Jackson and Heincke methods are of historical interest only and will not be considered here.

To illustrate the relations among the three estimators and to identify which of them is the best, each was applied to simulated data sets and to data from four real populations. A MonteCarlo simulation was used to compare the MLl estimator with the LS estimator. For analysis of catch curves, catch per unit of effort is assumed proportional to abundance, and under this assumption abundance and catch are interchangeable for the estimators considered in this study (i.e., mortality can be estimated with age-specific catch data).

\section{Notation}

For all of the methods, age is years after recruitment, where recruitment age is the age when the models first apply. Thus, rather than use terms such as $\left(y-y_{r}\right)$, where $y_{r}$ is recruitment age, age is denoted simply as $y$, having already been adjusted by subtraction of recruitment age. The following notation is used:

$x=$ nominal age, the age adjusted for recruitment assigned to an individual when the actual age adjusted for recruitment is between $x$ and $x+1$;

$y=$ true age of an individual in terms of years after recruitment;

$w=$ age of an individual at death in terms of years after recruitment;

$N_{x}=$ number of individuals in population or catch between age $x$ and $x+1$;

$N(y)=$ number of individuals in population or catch of exactly age $y$ for continuoustime models;

$N_{y}=$ number of individuals or catch of exactly age $y$ for discrete-time models;

$N=$ total size of recruited population or total catch;

$k=$ number of age groups in the recruited population minus one; 
$s=$ survival rate, the proportion of individuals alive at age $y$ that survive to age $y+1$

$a=$ annual mortality rate $=1-s$;

$Z=$ total instantaneous mortality coefficient;

$D_{x}=$ number of deaths between age $x$ and $x+1$

$m=$ number of deaths observed or calculated to have occurred.

\section{Discrete-Time Model}

Mortality estimators have been based on both discrete- and continuous-time models. The estimator developed by Chapman and Robson (1960) is based on the discrete-time survival equation

$$
N_{y+1}=s N_{y} .
$$

Survival from one age to the next is assumed constant. Applying equation (1) iteratively gives

$$
N_{y}=s N_{y-1}=s^{2} N_{y-2}=\ldots=s^{y} N_{0} .
$$

The proportion of the population of age $y$ is

$$
\frac{N_{y}}{N}=\frac{N_{0} s^{y}}{N}
$$

and because survival can be written as $s=1-$ $N_{0} / N$, the proportion of age- $y$ fish can be written as

$$
\frac{N_{y}}{N}=(1-s) s^{y}=a(1-a)^{y} .
$$

Equation (4) is the probability mass function of the geometric distribution. The ML estimator of survival obtained with this model is slightly biased; an unbiased estimator is (Chapman and Robson 1960)

$$
\hat{s}=\frac{\sum_{i=1}^{N} y_{i}}{N+\sum_{i=1}^{N} y_{i}-1} .
$$

The number of individuals that survive to exactly age $y$ is unknown, so to apply the Chapman and Robson (1960) estimator the approximations

$$
N=\sum_{x=0}^{k} N_{x}
$$

and

$$
\sum_{i=1}^{N} y_{i}=\sum_{x=1}^{k} x N_{x}
$$

are applied.

Discrete-time models give estimates of survival; estimates of the total instantaneous mortality coefficient are obtained from a relation between the continuous- and discrete-time models. A slightly biased estimator of $Z$ is (Chapman and Robson 1960)

$$
\hat{Z}=-\log _{e} \hat{s} \text {. }
$$

For large sample sizes, such as those obtained in fishery assessment, the variance of $\hat{s}$ can be estimated as (Chapman and Robson 1960)

$$
\hat{V}(\hat{s})=\frac{\hat{s}(1-\hat{s})^{2}}{N},
$$

and from the law of propagation of errors the estimator of $V(\hat{Z})$ is

$$
\hat{V}(\hat{Z})=\hat{V}(\hat{s}) / \hat{s}^{2} .
$$

Least squares also can be applied to the discrete-time survival equation (equation 1) for estimation of either the survival or mortality parameter. Application of the logarithm transformation to equation (2) and substitution of nominal ages for actual ages gives

$$
\log _{e} N_{x}=\log _{e} N_{0}+\left(\log _{e} s\right) x,
$$

and this can be written as

$$
u_{x}=\log _{e} N_{0}-Z x \text {, }
$$

where $u_{x}=\log _{e} N_{x}$ and $Z=-\log _{e} s$. It is well known that the LS estimator of $Z$ is

$$
\hat{Z}=\frac{\sum_{x=0}^{k}(x-\bar{x})\left(u_{x}-\bar{u}\right)}{\sum_{x=0}^{k}(x-\bar{x})^{2}},
$$

and the variance of $\bar{Z}$ is estimated as

$$
\hat{V}(\hat{Z})=\frac{\sum_{x=0}^{k}\left(u_{x}-\bar{u}\right)^{2}-\frac{\sum_{x=0}^{k}(x-\bar{x})\left(u_{x}-\bar{u}\right)^{2}}{\sum_{x=0}^{k}(x-\bar{x})^{2}}}{(k-1) \sum_{x=0}^{k}(x-\bar{x})^{2}} .
$$




\section{Continuous-Time Model}

The conventional approach for modelling mortality with a continuous-time equation is to assume mortality is described by the deterministic model

$$
\frac{d N(y)}{d y}=-Z N(y), \quad y>y_{r}
$$

The solution to equation (14) with the initial value $N(y)=N(0)$ at recruitment is

$$
N(y)=N(0) \exp (-Z y),
$$

which can be linearized and then least squares can be applied for estimation of $Z$ and $V(\hat{Z})$; this leads to equations (12) and (13).

Application of the exponential distribution, a continuous-probability distribution, to describe mortality gives the simple equation suggested by Ssentongo and Larkin (1973). The exponential death density function is

$$
f(w)=Z \exp (-Z w), \quad w>0,
$$

where $f(w)$ is the probability density of death at exactly age $w$. The expected age at death and the variance of $w$ are $E(w)=1 / Z$ and $V(w)=1 / Z^{2}$. The ML2 estimator of $Z$ is

$$
\hat{z}=\frac{m}{\sum_{i=1}^{m} w_{i}} .
$$

It has an insignificant bias. The ages at death are unknown in fishery assessment and the approximations

$$
\begin{aligned}
m & =\sum_{x=0}^{k} D_{x} \\
& =\sum_{x=0}^{k}\left(N_{x}-N_{x+1}\right)
\end{aligned}
$$

and

$$
\begin{aligned}
\sum_{i=1}^{m} w_{i} & =\sum_{x=0}^{k} x D_{x} \\
& =\sum_{x=0}^{k} x\left(N_{x}-N_{x+1}\right)
\end{aligned}
$$

are applied. For large values of $m$, the estimator of the variance of $\hat{Z}$ is

$$
\hat{V}(\hat{Z})=\hat{Z}^{2} / m \text {. }
$$

The exponential death-density function is an exponential relation between age and the number of survivors. The probability of survival to age $y$ is

$$
\begin{aligned}
P(y) & \stackrel{\bullet}{=} \int_{y}^{\infty} f(v) d v \\
& =\exp (-Z y)
\end{aligned}
$$

where $v$ is an integration variable; hence, for the exponential distribution, the number of individuals of age $y$ can be calculated with equation (15).

\section{Discussion}

All three of the mortality estimators are based on the same or closely related models and, given appropriate data, should give similar estimates for total mortality. However, data requirements for the three methods are quite different. The ML2 estimator requires the number of deaths at exactly age $w$. The ML1 estimator and the LS estimator can be applied with the number of individuals alive at exactly age $y$, but they both also can be applied with nominal age data, which are the only data available for fisheries (Beverton and Holt 1956).

To evaluate and compare the methods, I first applied them to known age structures calculated with the equation $N(y)=1,000 \exp (-Z y)$ to determine how well they recovered known values of $Z$. The LS estimator and the ML1 estimator recovered the exact values of $Z$ but the ML2 estimator underestimated the correct values. For the ML2 estimator the true and estimated values of $Z$ were:

$$
\begin{array}{llllll}
Z & 0.10 & 0.50 & 0.75 & 1.00 & 1.50 \\
\hat{Z} & 0.10 & 0.40 & 0.53 & 0.64 & 0.72
\end{array}
$$

If the exact ages at death for fish were known, as they are for laboratory experiments and studies of humans where the exponential distribution has often been applied, the ML2 estimator would be unbiased. But with data available for fisheries it is necessary to assume that individuals live to the end of the time interval in which they die and this results in an underestimate of mortality. The error is more serious with high mortality because then the number of deaths increases rapidly with age.

The estimators were applied to data for North Sea plaice Pleuronectes platessa (Gulland 1969), 
TABLE 1.-Comparison of mortality estimates $\hat{\mathbf{Z}}$ and their variances $\hat{\mathbf{V}}(\hat{\mathbf{Z}})$ generated by least - squares (LS), maximum-likelihood geometric (ML1), and maximum-likelihood exponential (ML2) models applied to four published data sets; $\mathrm{x}$ is years after recruitment.

\begin{tabular}{|c|c|c|c|c|c|c|c|c|c|c|c|c|}
\hline \multirow{2}{*}{$\begin{array}{c}\text { Observed } \\
\text { age } \\
\text { (years) }\end{array}$} & \multicolumn{3}{|c|}{ Plaice $^{\mathrm{a}}$} & \multicolumn{3}{|c|}{ Smallmouth bass ${ }^{b}$} & \multicolumn{3}{|c|}{ Pacific herring $^{c}$} & \multicolumn{3}{|c|}{ English sole ${ }^{d}$} \\
\hline & $x$ & Catch & Deaths & $x$ & Catch & Deaths & $x$ & Catch & Deaths & $x$ & Catch & Deaths \\
\hline 1 & & & & & 1 & & & 34 & & & & \\
\hline 2 & & 98 & & & 23 & & & 608 & & & & \\
\hline 3 & & 959 & & & 12 & & & 6,141 & & & & \\
\hline 4 & & 1,919 & & & 51 & & 0 & 2,607 & 0 & & 34 & \\
\hline 5 & 0 & 1,670 & 0 & & 146 & & 1 & 497 & 2,110 & 0 & 138 & 0 \\
\hline 6 & 1 & 951 & 719 & 0 & 84 & 0 & 2 & 91 & 406 & 1 & 77 & 61 \\
\hline 7 & 2 & 548 & 403 & 1 & 18 & 66 & 3 & 17 & 74 & 2 & 27 & 50 \\
\hline 8 & 3 & 316 & 232 & 2 & 5 & 13 & 4 & 4 & 13 & 3 & 11 & 16 \\
\hline 9 & 4 & 180 & 136 & 3 & 2 & 3 & 5 & 1 & 3 & 4 & 3 & 8 \\
\hline 10 & 5 & 105 & 75 & & & & & & & & & \\
\hline LS & \multicolumn{3}{|c|}{$\begin{aligned} \hat{Z} & =0.56 \\
\hat{V}(\hat{Z}) & =1.50 \times 10^{-6}\end{aligned}$} & \multicolumn{3}{|c|}{$\begin{aligned} \hat{Z} & =1.24 \\
\hat{V}(\hat{Z}) & =4.89 \times 10^{-3}\end{aligned}$} & \multicolumn{3}{|c|}{$\begin{aligned} \hat{Z} & =1.58 \\
\hat{V}(\hat{Z}) & =7.27 \times 10^{-4}\end{aligned}$} & \multicolumn{3}{|c|}{$\begin{aligned} \hat{Z} & =0.96 \\
\hat{V}(\hat{Z}) & =2.74 \times 10^{-3}\end{aligned}$} \\
\hline ML1 & \multicolumn{3}{|c|}{$\begin{aligned} \hat{Z} & =0.64 \\
\hat{V}(\hat{Z}) & =1.11 \times 10^{-4}\end{aligned}$} & \multicolumn{3}{|c|}{$\begin{aligned} \hat{Z} & =1.43 \\
\hat{V}(\hat{Z}) & =0.02\end{aligned}$} & \multicolumn{3}{|c|}{$\begin{aligned} \hat{Z} & =1.66 \\
\hat{V}(\hat{Z}) & =1.07 \times 10^{-3}\end{aligned}$} & \multicolumn{3}{|c|}{$\begin{aligned} \hat{Z} & =0.90 \\
\hat{V}(\hat{Z}) & =3.34 \times 10^{-3}\end{aligned}$} \\
\hline ML2 & \multicolumn{3}{|c|}{$\begin{aligned} \hat{Z} & =0.50 \\
\hat{V}(\hat{Z}) & =1.59 \times 10^{-4}\end{aligned}$} & \multicolumn{3}{|c|}{$\begin{aligned} \hat{Z} & =0.81 \\
\hat{V}(\hat{Z}) & =8.03 \times 10^{-3}\end{aligned}$} & \multicolumn{3}{|c|}{$\begin{aligned} \hat{Z} & =0.81 \\
\hat{V}(\hat{Z}) & =2.52 \times 10^{-4}\end{aligned}$} & \multicolumn{3}{|c|}{$\begin{aligned} \hat{Z} & =0.56 \\
\hat{V}(\hat{Z}) & =0.002\end{aligned}$} \\
\hline
\end{tabular}

a Number of plaice landed at Lowestoft per 100 hours of fishing (Gulland 1969).

b Catch of smallmouth bass in northern Lake Michigan (Latta 1957).

c Catch of Pacific herring off British Columbia (Tester 1955).

d Catch of English sole (Ricker 1958, who called the species lemon sole).

smallmouth bass Micropterus dolomieui (Latta 1957), Pacific herring Clupea harengus pallasi (Tester 1955), and English sole Parophrys vetulus (Ricker 1958). In every case, the ML2 estimator is below the others and the higher the value of $\hat{Z}$, the larger the difference (Table 1). The LS estimator is always the most precise.

To compare the LS estimator with the ML1 estimator, both were applied to calculate $\hat{Z}$ forage structures generated with the equation

$$
N(y)=N(0) \exp (-Z y+\xi),
$$

where $\xi$ is a normal random variable with a mean of zero. The instantaneous total mortality coefficient was one. The above equation is the con- ventional equation applied to estimate the mortality coefficient with regression analysis. For different standard deviations and different sample sizes, age structures were generated 1,000 times and the average $\hat{Z}$ and average variance of $\hat{Z}$ were calculated (Table 2 ). In all cases the average $\hat{Z}$ was very close to the true value. If the level of variation was small, the LS estimator was much more precise than the ML1 estimator, but as variation increased, the MLl estimator became more precise than the LS estimator ( $\mathrm{Ta}$ ble 2).

The number of animals observed has a large effect on the variance of the MLl estimator but no effect on the variance of the LS estimator

TABLE 2.-Average variances of mortality estimates based on 1,000 trials for least-squares (LS) and maximumlikelihood geometric (MLI) models with different levels of variation in the survival equation and different sample sizes.

\begin{tabular}{|c|c|c|c|c|c|c|}
\hline \multirow{3}{*}{$\begin{array}{l}\text { Survival } \\
\text { variance }\end{array}$} & \multicolumn{6}{|c|}{ Number of fish observed } \\
\hline & \multicolumn{2}{|c|}{100} & \multicolumn{2}{|c|}{500} & \multicolumn{2}{|c|}{1,000} \\
\hline & LS & ML1 & LS & ML1 & LS & ML1 \\
\hline 0.01 & $3.04 \times 10^{-6}$ & $7.06 \times 10^{-3}$ & $2.99 \times 10^{-6}$ & $1.42 \times 10^{-3}$ & $3.00 \times 10^{-6}$ & $7.12 \times 10^{-4}$ \\
\hline 0.05 & $7.70 \times 10^{-5}$ & $7.08 \times 10^{-3}$ & $7.62 \times 10^{-5}$ & $1.42 \times 10^{-3}$ & $7.70 \times 10^{-5}$ & $7.11 \times 10^{-4}$ \\
\hline 0.10 & $2.99 \times 10^{-4}$ & $7.12 \times 10^{-3}$ & $3.08 \times 10^{-4}$ & $1.43 \times 10^{-3}$ & $3.07 \times 10^{-4}$ & $7.13 \times 10^{-4}$ \\
\hline 0.50 & $7.50 \times 10^{-3}$ & $8.55 \times 10^{-3}$ & $7.58 \times 10^{-3}$ & $1.68 \times 10^{-3}$ & $7.51 \times 10^{-3}$ & $7.91 \times 10^{-4}$ \\
\hline 1.00 & 0.03 & 0.013 & 0.031 & $2.61 \times 10^{-3}$ & 0.03 & $1.36 \times 10^{-3}$ \\
\hline 1.25 & 0.048 & 0.021 & 0.047 & $3.32 \times 10^{-3}$ & 0.048 & $2.11 \times 10^{-3}$ \\
\hline
\end{tabular}


(Table 2); the precision of the ML1 estimator is a function of the number of animals whereas the precision of the LS estimator is a function of the number of age groups.

\section{References}

Beverton, R. J. H., AND S. J. Holt. 1956. A review of methods for estimating mortality rates in exploited fish populations, with special reference to sources of bias in catch sampling. Rapports et Procès-Verbaux des Réunions, Conseil International pour l'Exploration de la Mer 40:67-83.

Chapman, D. G., AND D. S. Robson. 1960. The analysis of a catch curve. Biometrics 16:354-368.

Gulland, J. A. 1969. Manual of methods for fish stock assessment. Part I. Fish population analysis. FAO (Food and Agriculture Organization of the United Nations) Manuals in Fisheries Science 4: $1-154$.

HEINCKE, F. 1913. Investigations on the plaice. General report 1: the plaice fishery and protective measures. Preliminary brief summary of the most important points of the report. Rapports et ProcèsVerbaux des Réunions, Conseil International pour l'Exploration de la Mer 16.
JACKSON, C. H. N. 1939. The analysis of an animal population. Journal of Animal Ecology 8:238-246.

LatTA, W. C. 1957. The life history of the smallmouth bass, Micropterus $d$. dolomieui, at Waugoshance Piont, Lake Michigan. Michigan Department of Conservation, Bulletin 5 of the Institute for Fisheries Research. Ann Arbor, MI, USA.

RICKER, W. E. 1958. Handbook of computations for biological statistics of fish populations. Fisheries Research Board of Canada Bulletin 119.

Robson, D. S., and D. G. Chapman. 1961. Catch curves and mortality rates. Transactions of the American Fisheries Society 90:181-189.

Seber, G. A. F. 1973. Estimation of animal abundance and related parameters. Griffin, London, England.

Ssentongo, G. W., AND P. A. Larkin. 1973. Some simple methods of estimating mortality rates of exploited fish populations. Journal of the Fisheries Research Board of Canada 30:695-698.

TESTER, A. L. 1955. Estimation of recruitment and natural mortality rate from age composition and catch data in British Columbia herring population. Journal of the Fisheries Research Board of Canada 12:649-681. 